\title{
PERCEPCIÓN DE LAS EMPRESAS ALIADAS A LA CORPORACIÓN UNIVERSITARIA RAFAEL NÚÑEZ
}

\author{
Paola Mouthon Ramos ${ }^{1}$ \\ Omaira Bernal Payares ${ }^{2}$ \\ Sandra Hernández Rodríguez ${ }^{3}$
}

\begin{abstract}
RESUMEN
El objetivo de este artículo es analizar el valor percibido por los aliados estratégicos de la Corporación Universitaria Rafael Núñez frente a los resultados obtenidos en el desarrollo de los convenios, con el fin de que se generen estrategias de marketing enfocadas a fortalecer las relaciones existentes y la creación de futuras alianzas, observando el valor percibido por las entidades en cuanto al desarrollo de la alianza y los beneficios mutuos logrados por el trabajo realizado, así como las consecuencias que las relaciones con el sector externo genera en la institución a corto, mediano y largo plazo, que permita el desarrollo de un plan de marketing para el fortalecimiento de las relaciones con el sector externo.
\end{abstract}

PALABRAS CLAVES

Estrategias de Marketing, plan de marketing, valor percibido.

\begin{abstract}
The goal of the present article is to analyze the value perceived by the strategic allies of the Corporación Universitaria Rafael Núñez in front of the results obtained in the development of the agreements in order to create marketing strategies aimed to strengthen existing relations and the creation of future alliances, by observing the perceived value of the entities as far as development of the alliance and the mutual benefits achieved by the word done, as well as with the consequences that the relations with the external sector creates in the corporation in a short, medium and long term goal, which allows the development of a marketing plan for the strengthening of the relations with the external sector.
\end{abstract}

\section{KEYWORDS}

Marketing Strategies, Marketing Plan, Perceived Value

Depositado en agosto 15 de 2014, aprobado en octubre 31 de 2014.

1 Administradora de Empresas de la Universidad de Cartagena, Colombia. Magister en Dirección de Marketing, Universidad Viña del Mar, Chile. Directora de la Maestría en Gestión Logística de la Escuela Naval Almirante Padilla. Miembro del Grupo de Investigación LOGER, Consultora Empresarial y Docente Universitaria. Email: paola.mouthon@gmail.com

2 Psicóloga de la Fundación Universitaria Católica del Norte, Colombia. Administradora en Servicios de la Salud - Universidad de Cartagena, Colombia-. Magíster en Administración - Universidad Nacional de Colombia-. Doctorante en Ciencias Sociales, Énfasis Gerencia -Universidad de Zulia, Venezuela-. Docente de tiempo completo de la Corporación Universitaria Rafael Núñez, Coordinadora de Área Profesional del Programa Administración de Empresas; y, Miembro del Grupo de Investigación Cartaciencia. Docente Universitaria. Email: Omaira.bernal@curnvirtual.edu.com

3 Administradora de Empresas de la UPTC, Colombia. - Magister en Dirección de Marketing de la Universidad Viña del Mar, Chile. Consultora en Gestión comercial de MiPymes, y Docente Universitaria.Email: smileh33@gmail.com 


\section{INTRODUCCIÓN}

La Corporación Universitaria Rafael Núñez (CURN), desde su concepción ha tenido presente el compromiso y la responsabilidad social con la sociedad, y desde su Misión, centrada en el Desarrollo Humano Sostenible, ha sembrado los principios filosóficos en la comunidad académica, para dar solución a esos problemas, respetando las libertades de los individuos.

La CURN, tiene como objetivo primordial lograr la articulación de los vínculos sociales y los intereses académicos; de tal manera, que la proyección social contribuya a la formación de los estudiantes de los diversos programas ofertados a la sociedad, favoreciendo la interacción entre la institución y diversos sectores sociales, elemento vital que cobra sentido en la razón misma de la sostenibilidad de la Corporación y su responsabilidad social.

La proyección social, se encuentra definida en el acuerdo 014 de 2009, de la corporación, como "la voluntad y decisión institucional de dirigirse e influir en su entorno impulsando los procesos académicos e investigativos desde su comunidad académica...., bajo los principios y lineamientos del desarrollo humano sostenible"(Pág.2), busca contribuir a sensibilizar y crear sentido de responsabilidad en los estudiantes acerca de las realidades que lo circundan, a forjarlo en valores como la solidaridad, la tolerancia, la equidad y el servicio, para que a través de ellos pueda visualizar el sentido de utilidad y aplicabilidad a lo aprendido, fortaleciendo a su quehacer profesional.

Para el desarrollo de sus actividades, cuenta con cuatro programas que le permiten servir a la comunidad cartagenera, así:

1. Prácticas Académicas y Formativas: organiza la participación del estudiante en un contexto determinado, en el cual tiene la oportunidad de afianzar y construir su propio conocimiento en el plano práctico. Se regulan a través de convenios.

2. Asesoría y Servicios: soporta la venta de servicios y asesorías especializadas a través de consultorios o centros constituidos por facultades afines, dirigidos por estudiantes de últimos semestres, docentes y egresados. Se regulan a través de contratos y convenios.

3. Educación Continuada: el Centro de Postgrados y Educación Continuada de la CURN, actúa como observatorio que identifica y da respuesta a las necesidades y requerimientos de formación del recurso humano del medio. Se realizan a través de contratos, alianzas y convenios.

4. UNYCO: sigla que conjuga la Universidad y la Comunidad, se instituyó con el fin de propiciar la identificación del ente académico con la comunidad; estrategia que posibilita la formación integral de los estudiantes con un sentido social.

El compromiso social de la CURN, señala las funciones sustantivas cotidianas que la comprometen: la docencia, la investigación y la proyección social, para la construcción de proyectos colectivos que generan sinergia y amplían la capacidad para servir mejor a la sociedad, en torno al logro de los Objetivos de Desarrollo del Milenio, en los 237 convenios vigentes, firmados con 186 empresas, que posee a Enero de 2013.

En estas empresas la CURN desarrolla sus programas de Proyección Social, constituyéndose una relación con el sector externo, que realiza a través de dos mecanismos: Alianzas, que se constituyen en relaciones a corto plazo para el logro de un programa o proyecto, se regulan a través de carta compromiso y Convenios, que se desarrollan con entidades que desean establecer relaciones a largo plazo.

En 1983, Berry utiliza el término de Marketing relacional manifestando que consiste en "atraer, mantener e intensificar las relaciones con los clientes". (Berry, 1983). Otro autor sostiene "uno de los aspectos más importantes y de mayor valor en una compañía son las relaciones que esta mantenga tanto con sus clientes como con sus proveedores, empleados, distribuidores y aliados" (Kotler, 2003). Entonces, su objetivo final es fidelizar al cliente, por medio de relaciones satisfactorias con los actores que intervienen en la empresa. Para lograr la fidelización 
deseada, es requerido un cambio estructural dentro de la organización, donde se encuentra la asimilación de conceptos como la calidad y el servicio, factores que afectan directamente la percepción y el valor percibido del cliente.

Al aplicar las cinco fuerzas de competitividad a la Corporación Universitaria Rafael Núñez, que enseñan que una empresa está rodeada de cinco factores fundamentales dentro de una industria y se debe aprender a controlarlos para sobrevivir en el mercado y tomar buenas decisiones. (Porter, 1980).

\section{MATERIALES Y MÉTODOS}

La presente investigación es de tipo descriptivo, utiliza el método inductivo, para determinar el valor percibido por las empresas, organizaciones y entidades en alianzas y convenios establecidos con la Corporación Universitaria Rafael Núñez, también utiliza para ello un enfoque cuantitativo, centrado en resultados estadísticos.

Para la recolección de información primaria se acudió a instrumentos para recolectar los datos como: Encuesta, realizada a las empresas, organizaciones y entidades con quienes la CURN tiene alianzas y convenios. Realizadas de forma virtual con el uso de herramientas informáticas de libre acceso, utilizando la página www.emarketing.com, complementado con seguimiento telefónico, con la finalidad de lograr el mayor número de respuestas posibles. Se realizaron Entrevistas con algunos directivos y responsables del manejo del convenio; se acudió a documentos propios de la institución como el Proyecto Educativo Institucional, documentos del Departamento de Proyección Social, entre otros. Para complementar se recurrió a textos, revistas e internet cuidando la fiabilidad de las fuentes consultadas.

La población objeto de estudios son los representantes de entidades con quienes la CURN tiene convenios vigentes. Número de convenios vigentes por programa a abril de 2013. En total la institución tiene convenios vigentes con 186 entidades. Con algunas de ellas se tiene dos tipos de convenios (por ejemplo de prácticas y de cooperación) y también una misma organiza- ción puede tener alianzas con varios programas. Bajo todo este panorama se procedió a calcular la muestra siguiendo los lineamientos de Fernández, (2002).

$$
\mathrm{n}=\mathrm{N} \mathrm{Z}^{2} \mathrm{P}^{*} \mathrm{Q} /(\mathrm{N}-1) \mathrm{e}^{2}+\mathrm{Z}^{2} \mathrm{P}^{*} \mathrm{Q} .
$$

Dando un resultado de la muestra (n) igual a 50 entidades.

La primera fase, recolección de información a través de fuentes primarias, como los datos suministrados por los representantes de las diversas entidades mediante encuestas sistematizadas y entrevistas a funcionarios de las mismas, cuya construcción se sustenta en la escala de PERVAL (Sweeney y Soutar, 2001). Este modelo plantea tres dimensiones básicas para ser estudiadas: Valor emocional, valor social y valor funcional.

La segunda fase, tabulación de los datos recolectados a través de la encuesta y las entrevistas no estructuradas, utilizando para ello herramientas electrónicas provistas por la página seleccionada para la realización del estudio y el análisis de datos extraídos de las entrevistas para complementar los resultados.

En la tercera fase, se analizaron los resultados los cuales serán presentados en el desarrollo del proyecto y sirvieron de base para formular conclusiones, estrategias y el plan de marketing como producto final.

\section{RESULTADOS}

Organismos no gubernamentales (ONG) y entidades sin ánimo de lucro con quienes la CURN realiza sus actividades de tipo social, constituyen el $19 \%$ de la totalidad de entidades con quienes la institución tiene convenios; El $9 \%$ está conformado por laboratorios clínicos donde interactúan programas de la salud como Bacteriología y Medicina, el 7\% por empresas del sector turístico de Cartagena de Indias, considerada Patrimonio Histórico y Cultural de la Humanidad, y un $12 \%$ por otro tipo de organizaciones como empresas contables y de sistemas. Cabe destacar que las entidades del estado fueron las menos receptivas para responder al 
instrumento de recolección de información, a pesar de que son el $26 \%$, solo el $2 \%$ atendió la solicitud. Estas entidades pueden tener suscritos con la CURN diferentes tipos de convenios dentro de los que se destacan:

CONVENIOS DE PRÁCTICAS ACADÉMICAS Y FORMATIVAS: Para que a través de los cuales los estudiantes de los diferentes programas puedan realizar sus prácticas empresariales, pedagógicas e investigativas, de cabina, de Docencia/Servicio (Área de la salud reguladas por el Decreto 2376 de 2010), comunitarias o sociales, entre otras. Determinadas en el Acuerdo 014 de 2009. Son considerados a largo plazo.

\section{CONVENIOS DE COOPERACIÓN INTER-} INSTITUCIONAL: Se suscriben para realizar actividades de Docencia, Investigación y Proyección Social, que beneficie a los actores y a una comunidad determinada. Se realizan brigadas, proyectos dentro de los programas: Escuela Saludable, Zona de Influencia y Gestión Social Corporativa, como Proyección Social interna de la entidad.

CONVENIOS ESPECÍFICOS: Se realizan con un programa para situaciones o proyectos puntuales. A través de ALIANZAS, mediante actas de trabajo y cartas de compromisos para realizar actividades puntuales de corto plazo sin suscribir convenios con la CURN.

En cuanto a las actividades que las entidades realizan con la CURN en desarrollo del objeto de los convenios, el 49\% están suscritos para el ejercicio de Prácticas Académicas y Formativas, seguido de un $10 \%$ de brigadas y $9 \%$ de proyectos conjuntos, enmarcados en convenios de cooperación institucional.

Teniendo en cuenta que la Escala de PERVAL contempla tres aspectos: Emocional, Social y Funcional, se analizaron los datos para determinar el valor percibido con respecto al desarrollo de los convenios suscritos con la CURN.

VALOR EMOCIONAL. Sentimientos que la prestación del servicio genera en los usuarios que se benefician en desarrollo del objeto de los convenios suscritos. El trato recibido por los funcionarios de la CURN en el desarrollo de las actividades del convenio, más del 90\% de las entidades encuestadas consideran que el trato recibido es bueno o muy bueno, siendo una fortaleza para la entidad en su recurso humano como activo que genera una empatía con las empresas aliadas, generando vínculos de amistad.

En cuanto al nexo con la CURN el 48,8\% de las empresas encuestadas la perciben como un socio estratégico en las actividades realizadas. Sin embargo, un $34,9 \%$ la ve como un aliado que siempre brinda apoyo, una percepción de un "amigo" que brinda acompañamiento, lo cual es positivo dentro del valor emocional percibido. Un 9,3\% la percibe como una entidad con la que se tiene una relación, pero no es muy cercana a la empresa y el 7\% la ve como un proveedor de servicios y no se establece vínculo emocional. El 16,3\% no siente nexos "emocionales" con la institución, la consideran una entidad con quien existe un convenio, único lazo que las une.

La pregunta de cómo se perciben las relaciones entre las entidades que tienen suscrito el convenio, la percepción que los encuestados poseen de la CURN, el 60,5\% de los encuestados creen que la relación es buena, y puede fortalecerse, el $23,3 \%$ cree que la relación es la mejor. Un $14 \%$ de aliados piensan que se relacionarán mejor en el futuro y el 2,3\% cree que no hay una relación más allá de la alianza. Por lo que es necesario fortalecer el interés de los aliados en que esto se haga.

Esta misma pregunta se manejó en la entrevista estructurada para los funcionarios de la CURN encargados de los convenios. Con respuestas muy similares, el 65\% considera que las relaciones son buenas pero pueden fortalecerse; un $35 \%$ cree que son excelentes. Al comparar los resultados desde el punto de vista externo e interno, las tendencias son afines, internamente es mayor el porcentaje que piensa que son excelentes y ninguno cree que son regulares.

VALOR FUNCIONAL. Valor de uso y de cambio de la relación, es decir, la expectativa de reci- 
bir lo que se espera frente a lo que realmente se da. El 79,1\% de los aliados de la entidad, consideran que las actividades y proyectos desarrollados con la CURN han sido excelentes y aportan a su organización; ellos le ven el valor agregado a la relación. Un 18,6\% piensan que han sido buenas las actividades realizadas, no aportan al desarrollo de su organización, aspecto que debe ser revisado por la IE, y un 2,3\% no se aporta nada y la relación es regular; quien respondió de esta manera en la entrevista, tuvo inconveniente con los estudiantes en práctica y siente que la institución no respaldó a la empresa aliada.

Al preguntar el beneficio económico que la empresa aliada ha tenido como resultado del convenio, el $62,8 \%$ ha encontrado que el convenio realizado ha contribuido a disminuir costos de la organización y el 23,3\% considera que ha reportado beneficios económicos a la entidad aliada. Entonces, el valor funcional percibido por el sector externo es alto y supera el $80 \%$ de las expectativas de los aliados encuestados. Un $14 \%$ de entidades externas piensan que la CURN no ha contribuido en beneficios económicos ni en la disminución de costos de su organización en el desarrollo de las actividades suscritas en el convenio.

Al consultar la opinión de los funcionarios de la CURN encargados de la operación de los convenios y sus actividades frente al valor funcional percibido, se encontró que el 53\% considera que la CURN aporta al Desarrollo de la región frente a un $47 \%$ que cree que la CURN da mucho más de lo que recibe a cambio.

Aspecto que se puede entender teniendo en cuenta que es una entidad sin ánimo de lucro, con un enorme sentido de responsabilidad social frente a su entorno, como lo expresa su misión institucional.

Con relación a la percepción que tienen las entidades externas a la CURN sobre la contribución al mejoramiento socioeconómico de la ciudad, el $84 \%$ de la población encuestada considera que los convenios si han contribuido a esta meta, mientras que un $16 \%$ considera que no. Del $100 \%$ de los encuestados, solamente el $30,2 \%$ presentó una justificación del porque consideraba que se había generado un aporte socioeconómico a la ciudad, encontrando que el $61,5 \%$ concuerda en que el desarrollo de los convenios suscritos, permiten la formación integral de personas que van a contribuir al mejoramiento social y económico de la ciudad y de su propio nivel de vida. Un 30,8\% comenta que esta contribución se debe a una articulación en trabajos y acciones en pos de logro de objetivos y metas comunes.

VALOR SOCIAL: Este se refiere a la capacidad de aumentar el auto-concepto del beneficiario, a la contribución del convenio suscrito, al reconocimiento de la entidad y su aceptación en el entorno.

Al preguntar a los encuestados los beneficios obtenidos por haber suscrito el convenio con la CURN, se encontró que el 58\% de los encuestados creen que el convenio les ha permitido tener una mejor imagen corporativa en el entorno, redundando claramente en una percepción positiva del valor social. Un $26 \%$ manifiesta que gracias al convenio ha podido ganar nuevos aliados. El 16\% comentan que el convenio ha permitido realizar actividades conjuntas pero no ha afectado la imagen de la empresa en el entorno. Ninguno de los encuestados cree que los convenios hayan perjudicado la imagen de la empresa.

Cabe destacar que el 100\% de las entidades encuestadas manifestó que recomendarían a otras entidades realizar convenios con la Corporación Universitaria Rafael Núñez, siendo éste uno de los principales beneficios del valor social percibido.

En cuanto a las sugerencias para mejorar, por parte del sector externo, el $35.3 \%$ manifiesta que debería existir una comunicación más fluida entre la CURN y el aliado, al igual que tener en cuenta que debe existir retroalimentación a lo largo del convenio como al finalizar el mismo, para corregir y mejorar las fallas que puedan presentarse. El $20.6 \%$ de los aliados manifestaron la necesidad de hacer seguimiento a los estudiantes y al convenio, sugiriendo, incluso, la asignación de un tutor que nutra una comunicación constante entre las instituciones. 
El 8.82\% manifiesta que les gustaría la ampliación de los convenios a otros programas, mostrando esto una oportunidad de crecimiento; el $5.88 \%$ solicitan definir claramente el horario en el cual se realizarían las prácticas y sobre todo tener en cuenta el horario que le convenga al aliado; el $2.94 \%$ pide que se mantengan actualizados los convenios para solicitar el personal correcto de acuerdo a las necesidades que se tengan; el $2.94 \%$ pide tener en cuenta a los egresados para realizar las prácticas; otro $2.94 \%$ pide que se haga la teoría y la práctica al tiempo para que los estudiantes tengan frescos los temas, el $11.8 \%$ manifiesta que todo está bien y no tienen sugerencias, y, finalmente, un $8.82 \%$ tienen otras solicitudes diversas.

Las sugerencias para mejorar las relaciones con el sector externo, por parte de los funcionarios de la CURN, se centraron en solicitar convenios con mayor impacto y buscar ganancias por partes iguales en las actividades implementadas a lo largo del convenio.

En cuanto a los principales motivos de queja de las entidades aliadas que reciben los funcionarios de la CURN indagados durante la entrevista, se resalta que el $32 \%$ de las mismas se refieren a aspectos financieros relacionados con el manejo de convenios. Un $16 \%$ ha recibido quejas relacionadas con algún aspecto administrativo relacionado con el desarrollo del convenio y otro $16 \%$ se relaciona a comportamientos de estudiantes y/o docentes que generan molestias en los sitios, especialmente asociados al desarrollo de prácticas.

Es de resaltar que el $26 \%$ de los funcionarios encuestados NUNCA han recibido ninguna queja por parte de las entidades con quienes mantiene relación.

\section{DIAGNÓSTICO}

La Corporación Universitaria Rafael Núñez es una entidad de carácter privado que tiene gran fortaleza en el establecimiento de alianzas con entidades, fundaciones y organizaciones del sector productivo, especialmente, para el desarrollo de prácticas empresariales, pero también para el desarrollo de actividades que impactan a la ciudad y la región.
En el marco de estas alianzas genera un fuerte valor emocional, permitiendo que sus aliados lo perciban como el socio y amigo que los acompaña en sus actividades; sin embargo, también es necesario mejorar las relaciones que, si bien son buenas, hay que fortalecerlas mediante el seguimiento a las actividades de los convenios, canales de comunicación más efectivos, además de optimizar el flujo de procesos académicos y financieros en torno a los convenios.

El valor funcional generado frente a los aliados es alto, pues le reporta beneficios económicos y de impacto social; no obstante, los funcionarios de la entidad sienten que se da mucho a cambio de poco. Ambos actores coinciden en que la sinergia entre la universidad, el Estado y el sector productivo es necesaria para mejorar las condiciones socioeconómicas de la ciudad y la región. A pesar de la existencia de la competencia, en especial, en escenarios de prácticas, el trabajo realizado por la CURN, abre la puerta a gran cantidad de oportunidades para ampliar su accionar en el desarrollo de las alianzas.

En cuanto al valor social, la alianza con la CURN, le ha generado la posibilidad a los aliados de ser reconocidos socialmente y haber logrado otros nexos, lo que genera oportunidades de crecimiento, porque el $100 \%$ de los encuestados recomendaría a otras entidades establecer convenios con la entidad.

\section{PLAN DE MARKETING}

Análisis de la situación: La Corporación Universitaria Rafael Núñez, como institución de educación superior de carácter privado, tiene como parte fundamental de su misión, la formación integral de seres humanos que como profesionales tengan la oportunidad de contribuir con la transformación socio cultural de su entorno local y regional, a través de principios de desarrollo humano sostenible y calidad.

Para ello, la institución, ha generado alianzas estratégicas a través de convenios que han facilitado esta labor significativamente. A través de un plan de marketing general, ejecutar estrategias que ayude no solo a mejorar puntos débiles encontrados en el estudio, sino a fortalecer las 
ventajas que por medio de los convenios se alcanzan, creando así un mayor valor corporativo ante la comunidad.

Hay muchos factores a mejorar en pos de fortalecer las relaciones entre la CURN y las instituciones aliadas. Existen oportunidades interesantes que se deben explorar y tomar con el propósito de fortalecer las relaciones existentes con los actuales aliados; debilidades que deben ser minimizadas con el fin de blindarse frente a los posibles competidores; amenazas que se deben atender para no dar pie a que otras entidades educativas de la ciudad o la región puedan tomar ventaja sobre la CURN y ofrezcan mejores opciones de alianzas y fortalezas que se deben utilizar para maximizar las cualidades que hacen de la CURN una institución de gran valor para la costa Caribe colombiana.

ANÁLISIS DOFA: con el fin de brindar un análisis más profundo de los datos recolectados. Éstos se clasificaron en debilidades y fortalezas, correspondiente a aspectos internos de la curn en desarrollo de los convenios y oportunidades y amenazas, relacionados con aspectos del entorno que la curn no puede cambiar $\mathrm{y}$ afectan el proceso, con el fin de poder plasmarlas en un análisis dofa, fundamental para el estudio realizado, que permita diseñar estrategias articuladas a las necesidades reales de los actores involucrados en el proceso.

\begin{tabular}{|c|c|c|c|}
\hline DEBILIDADES & FORTALEZAS & OPORTUNIDADES & AMENAZAS \\
\hline $\begin{array}{l}\text { D1: Convenios utilizados } \\
\text { para prácticas, en su mayoría, } \\
\text { desaprovechando otras actividades } \\
\text { de extensión e investigación. } \\
\text { D2: Existen entidades que ven a la } \\
\text { CURN como una entidad con quien } \\
\text { tienen convenios pero no sienten } \\
\text { conexión con ella. } \\
\text { D3: Los aliados y el personal de } \\
\text { la CURN coinciden en pensar que } \\
\text { aunque las relaciones con los aliados } \\
\text { son buenas, hay que fortalecerlas. } \\
\text { D4: Existen algunos aliados que } \\
\text { consideran que las actividades } \\
\text { realizadas con la CURN en el } \\
\text { desarrollo del convenio no aportan } \\
\text { mucho a la entidad como tal. } \\
\text { D5: Los funcionarios de la CURN } \\
\text { consideran que la institución recibe } \\
\text { muy poco a cambio en el desarrollo } \\
\text { de los convenios. } \\
\text { D6: Presupuesto limitado para las } \\
\text { actividades de Proyección Social. } \\
\text { D7: Quejas por manejos } \\
\text { administrativos y financieros de los } \\
\text { convenios. } \\
\text { D8: Falta seguimiento a las } \\
\text { actividades desarrolladas en los } \\
\text { convenios de acuerdo a percepción } \\
\text { de los aliados. } \\
\text { D9: Hace falta la presencia de la } \\
\text { CURN en eventos y actividades } \\
\text { de gran impacto a nivel nacional e } \\
\text { internacional. } \\
\text { D10: Ineficiente comunicación entre } \\
\text { la CURN y los aliados. }\end{array}$ & $\begin{array}{l}\text { F1: Excelente atención a los aliados por parte } \\
\text { del personal de la CURN. } \\
\text { F2: Equipo de trabajo serio y comprometido. } \\
\text { F3: Existencia de un nexo emocional de la } \\
\text { CURN con la mayoría de sus aliados. } \\
\text { F4: Gran número de convenios vigentes con } \\
\text { la CURN para el desarrollo de las actividades } \\
\text { de los Programas Académicos. } \\
\text { F5: Más del } 80 \% \text { de los aliados considera que } \\
\text { las actividades realizadas son buenas. } \\
\text { F6: Todos los encuestados coinciden en } \\
\text { afirmar que recomendarían a la CURN a otras } \\
\text { entidades para el desarrollo de convenios. } \\
\text { F7: Los convenios aportan al valor funcional } \\
\text { de los aliados, contribuyendo con beneficios } \\
\text { económicos a los mismos } \\
\text { F8: Se reconoce el Modelo Pedagógico de } \\
\text { la CURN como una entidad que sensibiliza } \\
\text { a sus estudiantes frente a las necesidades del } \\
\text { medio. } \\
\text { F9: Establecimiento de nexos de amistad } \\
\text { entre funcionarios CURN y personal de las } \\
\text { entidades aliadas, lo que facilita el manejo } \\
\text { del convenio. } \\
\text { F10: Existencia de bases de datos y } \\
\text { archivos fisicos y digitales muy completos y } \\
\text { organizados en relación a los convenios. } \\
\text { F11: Existencia de una estructura académico } \\
\text { administrativa adecuada para el manejo de } \\
\text { los convenios. } \\
\text { F12: Misión institucional centrada en el DHS } \\
\text { permite una gran sensibilidad social que } \\
\text { facilita la interacción con aliados. } \\
\text { F13. Generación de nuevos programas } \\
\text { académicos por parte de la entidad, que } \\
\text { responden a las necesidades de formación del } \\
\text { sector externo. } \\
\text { F14: La CURN ofrece servicios y asesorías } \\
\text { a través del Centro Empresarial, Clínica } \\
\text { Odontológica y Cabina de estética que le } \\
\text { permiten ampliar su radio de acción en el } \\
\text { desarrollo de convenios. } \\
\text { F15: Existencia de políticas claras de } \\
\text { Proyección Social que direccionan el trabajo } \\
\text { con aliados, a través del acuerdo } 014 \text { de } 2009 .\end{array}$ & $\begin{array}{l}\text { O1: Existe interés por parte de los } \\
\text { aliados en fortalecer las relaciones } \\
\text { con la CURN. } \\
\text { O2: Se reconoce a la CURN en } \\
\text { el medio como una entidad que } \\
\text { impacta socioeconómicamente a la } \\
\text { ciudad y la región. } \\
\text { O3: Percepción alta del valor social } \\
\text { de la CURN como una entidad que } \\
\text { mejora la imagen de sus aliados y } \\
\text { facilita el establecimiento de nuevas } \\
\text { alianzas. } \\
\text { O4: Deseo de los aliados de ampliar } \\
\text { el radio de acción de convenios } \\
\text { específicos a otros programas. } \\
\text { O5: Percepción de los aliados de la } \\
\text { CURN, a quien ven como un socio } \\
\text { y la entidad que los apoya cuando } \\
\text { lo necesitan. } \\
\text { O6: Alta posibilidad de lograr } \\
\text { alianzas que generen mayor impacto } \\
\text { para la ciudad. } \\
\text { O7: Concientización por parte del } \\
\text { sector empresarial y productivo de } \\
\text { la necesidad de generar alianzas con } \\
\text { entidades de Educación Superior, } \\
\text { para impactar en el medio. } \\
\text { O8: Existencia de bases de datos } \\
\text { de empresas a las que a través de } \\
\text { convenios se les puede ofrecer } \\
\text { educación continuada y postgrados. } \\
\text { O9: La CURN es líder en la } \\
\text { ciudad como ente de formación } \\
\text { en el área de salud, lo que le } \\
\text { brinda oportunidades únicas para } \\
\text { el desarrollo de alianzas que la } \\
\text { competencia no puede aprovechar. }\end{array}$ & $\begin{array}{l}\text { A1: Amplia Competencia de } \\
\text { otras entidades educativas en } \\
\text { la búsqueda de escenarios de } \\
\text { prácticas. } \\
\text { A2: Inseguridad reinante en } \\
\text { la ciudad afecta el desarrollo } \\
\text { de actividades en comunidad, } \\
\text { en el marco de los convenios } \\
\text { institucionales. } \\
\text { A3: El sector empresarial } \\
\text { cuenta con poco interés } \\
\text { de trabajar con otras } \\
\text { universidades, diferentes a } \\
\text { aquellas con las cuales ya } \\
\text { tienen acciones. } \\
\text { A5: La experiencia de otras } \\
\text { IES en la generación de } \\
\text { alianzas para el desarrollo de } \\
\text { proyectos de investigación y } \\
\text { participación en convocatorias } \\
\text { que brindan recursos para su } \\
\text { ejecución }\end{array}$ \\
\hline
\end{tabular}

Ilustración 1 Matriz DOFA 
Después de clasificar los diferentes factores encontrados, y de tener un panorama real de la situación actual con las diferentes empresas, se inicia el planteamiento de los objetivos y las estrategias. Estas últimas, son resultado del análisis y el cruce de cada una de las fortalezas, Amenazas, Debilidades y Oportunidades, y del análisis de las cinco fuerzas de Porter. La siguiente tabla muestra el ejercicio de elaboración de estrategias FO, que busca aprovechar las oportunidades a través de las fortalezas adquiridas, por el momento, se observa el cruce de las fortalezas 1 y 2 frente a las oportunidades 1 y 2 , generando como resultado unas estrategias F1O1 y F2O2.

\begin{tabular}{|c|c|c|}
\hline \begin{tabular}{|r|} 
FactoresExternos \\
\end{tabular} & \multirow{2}{*}{\multicolumn{2}{|c|}{ Lista de Oportunidades }} \\
\hline Factores Internos & & \\
\hline Lista de Fortalezas & $\begin{array}{l}\text { Ol: Existe interés por parte de los } \\
\text { aliados en fortalecer las relaciones con } \\
\text { la CURN. }\end{array}$ & $\begin{array}{l}\text { O2: Se reconoce a la CURN en el medio } \\
\text { como una entidad que impacta } \\
\text { socioeconómicamente a la ciudad y la } \\
\text { región. }\end{array}$ \\
\hline $\begin{array}{l}\text { F1: Excelente atención a los } \\
\text { aliados por parte del personal } \\
\text { de la CURN. }\end{array}$ & $\begin{array}{l}\text { F1O1 - Aprovechar la buena relacion } \\
\text { exisitente entre la CURN y sus aliados para } \\
\text { fortalecer la comunicación a traves de } \\
\text { boletines trimestrales que comenten los } \\
\text { logros de los convenios, los retos y } \\
\text { compromisos para superarlos. }\end{array}$ & \\
\hline $\begin{array}{l}\text { F2: Equipo de trabajo serio y } \\
\text { comprometido. }\end{array}$ & & $\begin{array}{l}\text { F2O2 - Realizar Eventos de socialización } \\
\text { académica en donde las empresas aliadas } \\
\text { puedan hablar de su misión y cómo los } \\
\text { convenios han ayudado a estas instituciones } \\
\text { a proyectarse socieconómicamente en la } \\
\text { región. }\end{array}$ \\
\hline
\end{tabular}

Ilustración 2 Estrategia FO

En el siguiente cuadro se ejemplifica el cruce de debilidades con amenazas, en donde se busca minimizar las debilidades encontradas, atacando a la vez las amenazas detectadas.

\begin{tabular}{|c|c|c|}
\hline Factores Externos & \multicolumn{2}{|c|}{ Lista de Amenazas } \\
\hline Lista de Debilidades & $\begin{array}{l}\text { A1: Amplia Competencia de otras entidades } \\
\text { educativas en la búsqueda de escenarios de } \\
\text { prácticas. }\end{array}$ & $\begin{array}{l}\text { A2: Inseguridad reinante en la ciudad afecta el } \\
\text { desarrollo de actividades en comunidad en el marco de } \\
\text { los convenios institucionales. }\end{array}$ \\
\hline $\begin{array}{l}\text { D1: Convenios utilizados } \\
\text { para prácticas en su } \\
\text { mayoria, } \\
\text { desaprovechando otras } \\
\text { actividades de extensión e } \\
\text { investigación. }\end{array}$ & $\begin{array}{l}\text { D1A1-Aprovechar la capacidad instalada de los sitios } \\
\text { para generar proyectos de investigación y asi destarcarse } \\
\text { en éste aspecto frente a la competencia. }\end{array}$ & $\begin{array}{l}\text { D1A2: Generar convenios con instituciones con alta } \\
\text { credibilidad social, que brinden seguridad como la Defensa } \\
\text { Civil, Policía Nacional, etc, para que conjuntamente se } \\
\text { elaboren proyectos que generen lazos con las comundades } \\
\text { con resultados medibles de promoción del desarrollo local y } \\
\text { la calidad de vida de los habitantes de la ciudad y la región. }\end{array}$ \\
\hline
\end{tabular}

Ilustración 3 Estrategia DA

En la siguiente imagen, se cruzan las debilidades con las oportunidades, con el fin de minimizar las debilidades halladas, aprovechando las oportunidades encontradas. Donde surgen estrategias 


\begin{tabular}{|c|c|c|}
\hline Factores Externos & \multirow{2}{*}{\multicolumn{2}{|c|}{ Lista de oportunidades }} \\
\hline Factores Internos & & \\
\hline Lista de Debilidades & $\begin{array}{l}\text { O1: Existe interés por parte de los aliados } \\
\text { en fortalecer las relaciones con la CURN. }\end{array}$ & $\begin{array}{l}\text { O2: Se reconoce a la CURN en el medio como una } \\
\text { entidad que impacta socioeconómicamente a la ciudad } \\
\text { y la región. }\end{array}$ \\
\hline $\begin{array}{l}\text { D1: Convenios utilizados para prácticas } \\
\text { en su mayoria, desaprovechando otras } \\
\text { actividades de extensión e } \\
\text { investigación. }\end{array}$ & 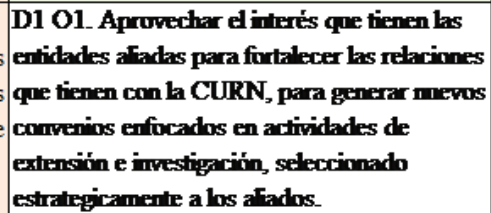 & $\begin{array}{l}\text { D1 02, Teniendo en coenta el reconociniento como una } \\
\text { entidad que imparta socineconímicamente a la cindad y a } \\
\text { la regón, se deberia apruvechar para generar comvenios de } \\
\text { investigarión que bencficie a ambas partes }\end{array}$ \\
\hline
\end{tabular}

Ilustración 4 Estrategia DO

El cruce final se hace entre las fortalezas y las amenazas, con el fin de encontrar formas de contrarrestar las amenazas detectadas a través de las fortalezas de la CURN y de esta manera, estar atentas a posibles situaciones externas que pueden afectar la labor realizada.

\begin{tabular}{|c|c|c|}
\hline Factores Externos & \multirow{2}{*}{\multicolumn{2}{|c|}{ Lista de Amenazas }} \\
\hline Factores Internos & & \\
\hline Lista de Fortalezas & $\begin{array}{l}\text { Al: Amplia Competencia de otras } \\
\text { entidades educativas en la búsqueda de } \\
\text { escenarios de prácticas. }\end{array}$ & $\begin{array}{l}\text { A2: Inseguridad reinante en la ciudad } \\
\text { afecta el desarrollo de actividades en } \\
\text { comunidad en el marco de los convenios } \\
\text { institucionales. }\end{array}$ \\
\hline $\begin{array}{l}\text { F2: Equipo de trabajo serio y } \\
\text { comprometido. }\end{array}$ & 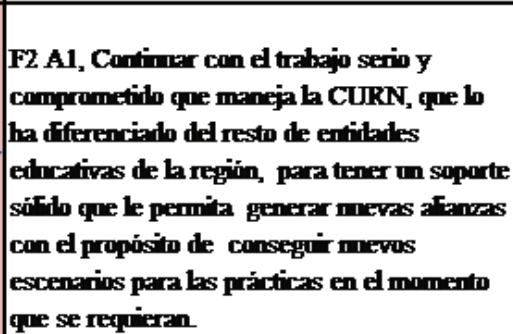 & 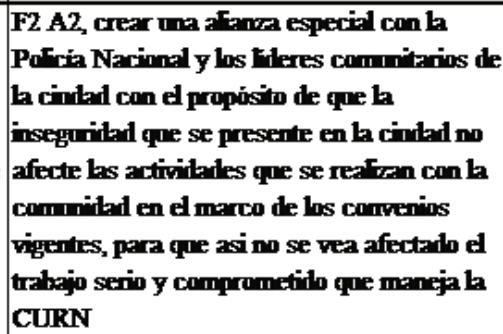 \\
\hline
\end{tabular}

Ilustración 5 Estrategia FA

Objetivos del plan de Marketing: Con el fin de tener una concordancia importante entre los objetivos del Plan de Marketing y las metas y planes de la Corporación Universitaria Rafael Núñez, se realizó un comité entre la universidad y el grupo investigador, en donde se discutieron los objetivos General y estratégicos.

\section{Objetivo General}

Afianzar la relación existente entre la Corporación Universitaria Rafael Núñez y las empresas aliadas a través de los convenios existentes y futuros.

\section{Objetivos estratégicos}

- Fortalecer la comunicación con las entidades aliadas y cooperantes para el manteni- miento de una retroalimentación constante, que permita el establecimiento de acciones oportunas con el sector externo.

- Diseñar, ejecutar y evaluar portafolios de servicios orientados a las necesidades reales del sector productivo que desde la academia puedan atenderse.

- Generar espacios académicos donde los funcionarios de la CURN y las entidades aliadas depositen ideas de fortalecimiento mutuo, de tal manera que haya una mayor ganancia para las partes

- Desarrollar proyectos de investigación con el sector externo que generen nuevos conocimientos e impacten positivamente a la región Caribe.

- Fortalecer las relaciones existentes con el 
sector externo, donde se manejen semilleros de investigadores y de Gestores Sociales, y se brinde un valor agregado a la relación.

Estrategias: Se realizó una selección, teniendo en cuenta las prioridades institucionales establecidas y la escala de Perval; Entonces la valoración de las estrategias desde el punto de vista del valor afectado, es decir, las estrategias relacionadas a los lazos de afecto que se han construido entre las dos instituciones, se seleccionaron como estrategias de valor emocional, pues su objeto es fortalecer esta percepción entre las dos instituciones. A continuación se muestra con mayor claridad la selección realizada:

Estrategias de valor emocional

Como estrategias de valor emocional se seleccionaron las siguientes:

\begin{tabular}{|c|c|c|c|c|c|c|}
\hline \multirow{2}{*}{\begin{tabular}{|c|} 
Ponderaciones \\
Estrategias
\end{tabular}} & \multirow{2}{*}{$\begin{array}{r}1 \text { menos valor - } 5 \text { el mayor valor } \\
\text { Descripcion }\end{array}$} & \multicolumn{5}{|c|}{ mportancia para la CURN y ALIAD } \\
\hline & & 1 & 2 & 3 & 4 & 5 \\
\hline \multirow{7}{*}{$\begin{array}{l}\text { VALOR } \\
\text { EMOCIONAL }\end{array}$} & 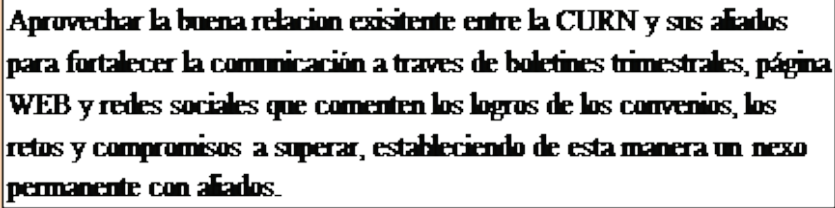 & & & & & $\mathbf{x}$ \\
\hline & 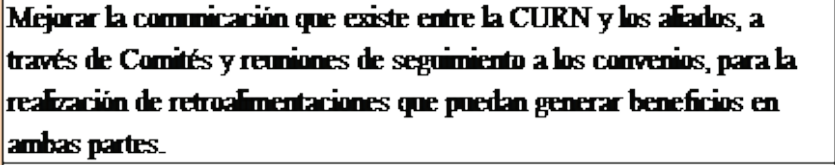 & & & & & $\mathbf{x}$ \\
\hline & 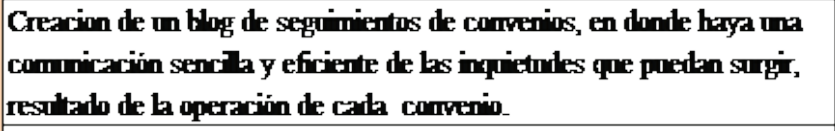 & & & & $\mathbf{x}$ & \\
\hline & $\begin{array}{l}\text { Aprovechar el nexo emocinal con aralos, rearyar activilades de } \\
\text { mayor inpacto social }\end{array}$ & & & & & $\mathbf{x}$ \\
\hline & 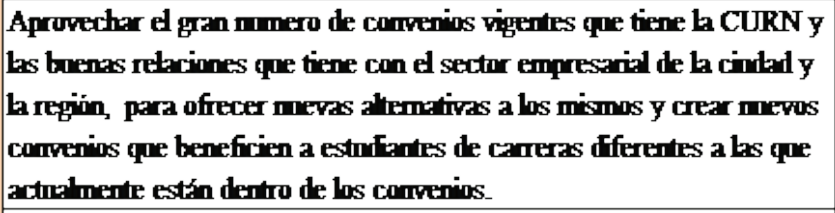 & & & & $\mathbf{x}$ & \\
\hline & 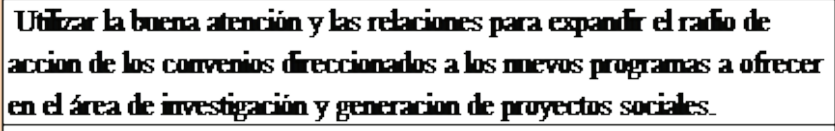 & & & & & $\mathbf{x}$ \\
\hline & 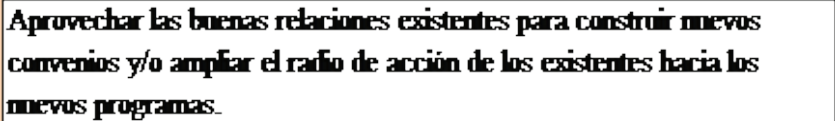 & & & & & \\
\hline
\end{tabular}

Ilustración 6 Estrategia del valor Emocional 


\section{Estrategias de valor social}

Como estrategias de valor social se seleccionaron las siguientes:

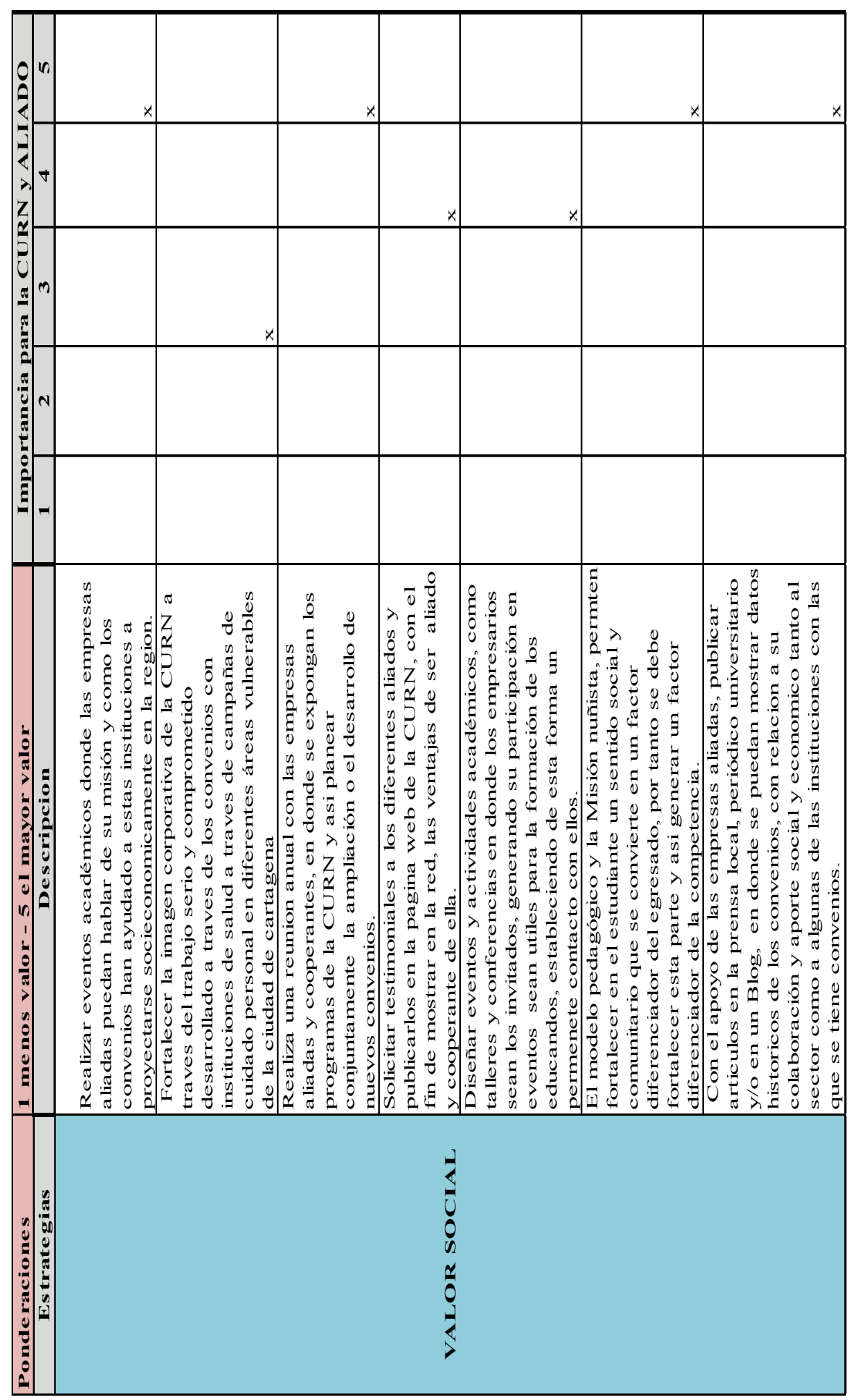

Ilustración 7 Estrategia de valor social 
Estrategias de valor funcional

Como estrategias de valor funcional se seleccionaron

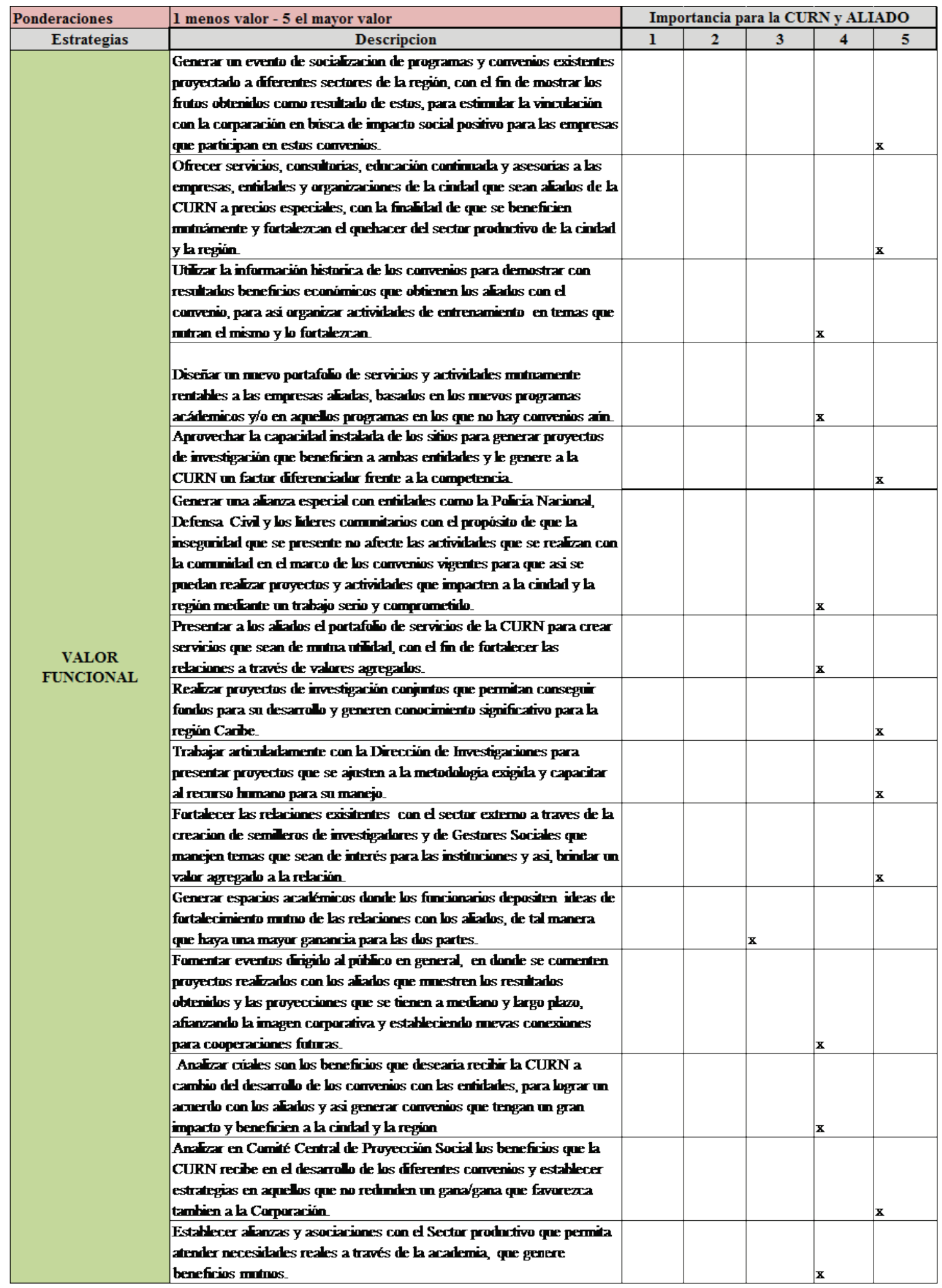




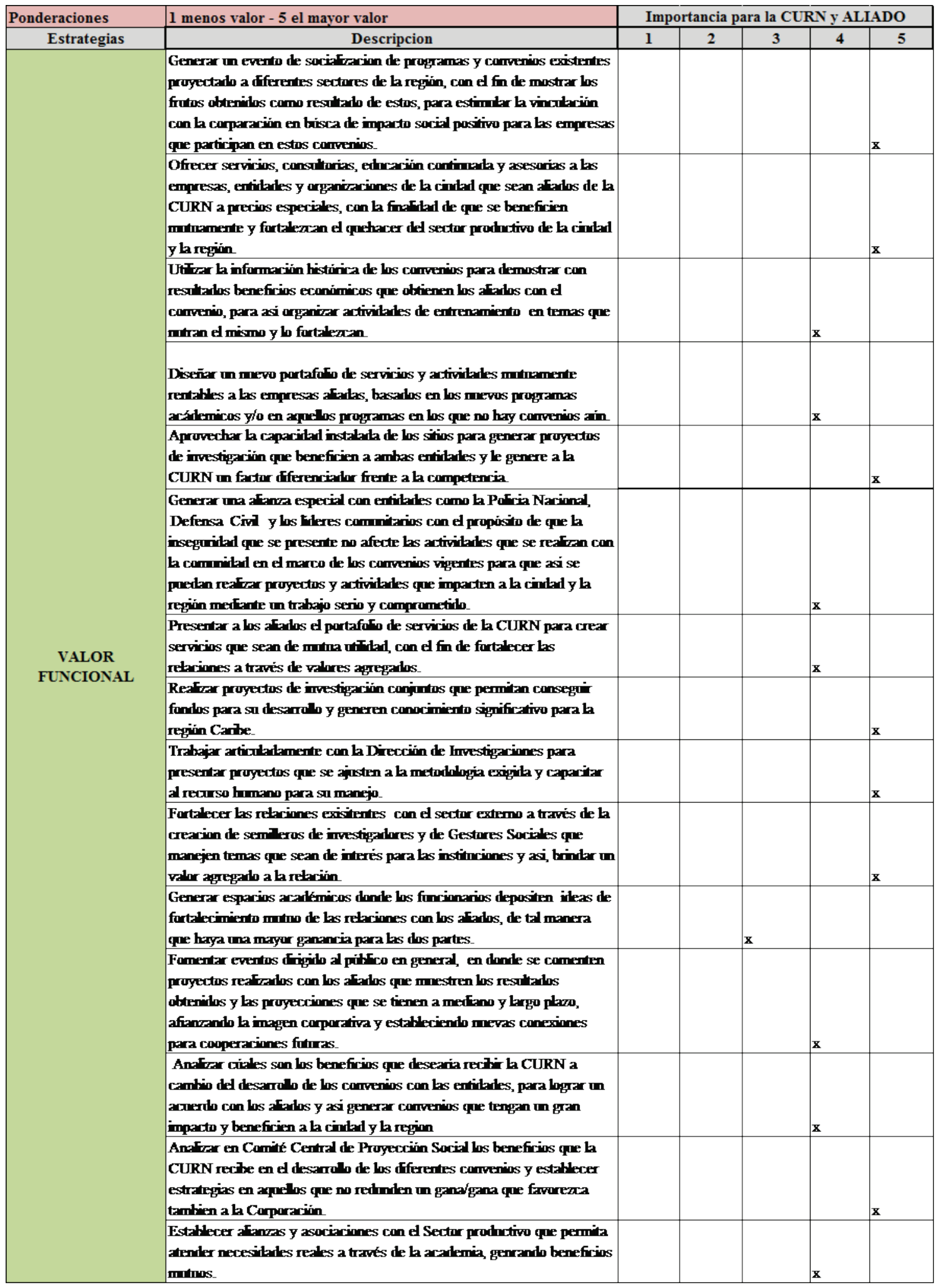

Ilustración 8 Estrategia del Valor Funcional 


\section{Plan de acción}

\begin{tabular}{|c|c|c|c|c|c|c|c|c|c|c|c|c|c|c|c|}
\hline \multicolumn{16}{|c|}{$\begin{array}{c}\text { DIRECCIÓN DE PROYECCIÓN SOCIAL } \\
\text { PLAN DE ACCIÓN RELACIONES CON EL SECTOR EXTERNO }\end{array}$} \\
\hline & & & \multirow{2}{*}{\multicolumn{3}{|c|}{ Denominacion p }} & ramas & Académ & cos: & & & & & & & \\
\hline \multirow{2}{*}{ Lineas Estratégicas } & \multirow{2}{*}{ OBJETIVOS } & \multirow{2}{*}{ ACCIONES } & & & & \multicolumn{2}{|c|}{ Responsabole } & \multirow{2}{*}{ 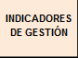 } & \multicolumn{2}{|c|}{ RECURSOS } & \multicolumn{2}{|c|}{ PLAZO } & \multirow{2}{*}{$\begin{array}{l}\text { FFCCAADE } \\
\text { CORIE }\end{array}$} & \multirow{2}{*}{ 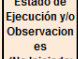 } & \multirow{2}{*}{ 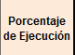 } \\
\hline & & & $\mathrm{CP}$ & MP & LP & ALLADO & $\mathbb{E E S}$ & & FIIICOS & Huмамоs & $\begin{array}{l}\text { Fochadod } \\
\text { thicio }\end{array}$ & 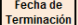 & & & \\
\hline \multirow{3}{*}{ 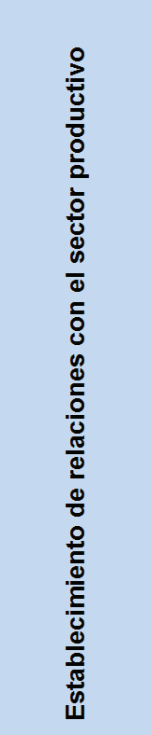 } & \multirow{3}{*}{ 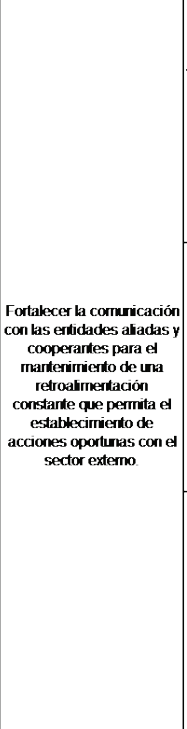 } & 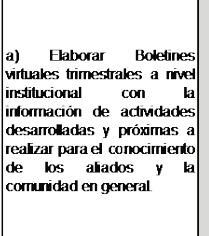 & & K & & & 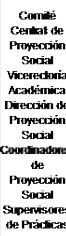 & 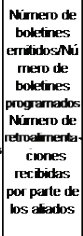 & 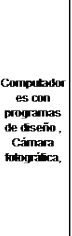 & 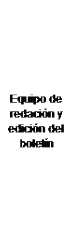 & Hito de 2013 & Husto de 2015 & semestar & & \\
\hline & & 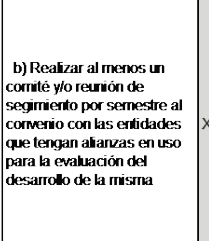 & & & & 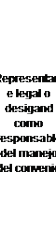 & 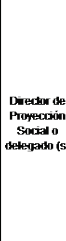 & 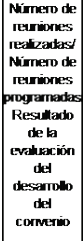 & 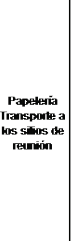 & 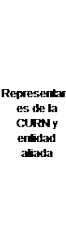 & 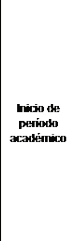 & 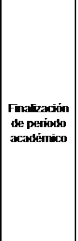 & senestat & & \\
\hline & & 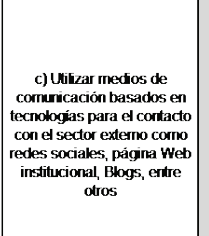 & & $x$ & & & 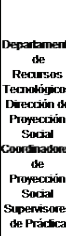 & 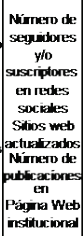 & 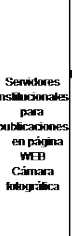 & 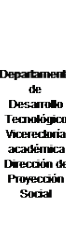 & 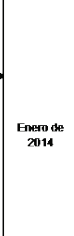 & 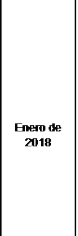 & mesesen & & \\
\hline
\end{tabular}

Ilustración 9 Plan de Acción

\section{CONCLUSIONES}

Desarrollado el presente estudio, se puede concluir que la Corporación Universitaria Rafael Núñez goza de una buena imagen institucional ante sus aliados del sector externo, reflejada en el valor emocional, generando con ello el desarrollo de las actividades derivadas de la relación.

Los aliados también perciben en la mayoría de los casos el valor social que ha generado la relación al permitirle lograr otras alianzas o ganar "status" en el mercado, lo que podría ayudar a la Corporación en la consecución de nuevos aliados,

El valor funcional es alto, aspecto que se debe tener presente. Es importante resaltar que los funcionarios de la CURN sienten que dan más de lo que se recibe a cambio, lo cual es lógico por la razón social de la corporación y la misión centrada al desarrollo humano sostenible.

Queda demostrado también la importancia que representa la relación con el sector externo para la institución, no solo porque se constituye en la fortaleza de la CURN sino porque gracias a esa interrelación es posible retroalimentar currículos, desarrollar portafolios de servicios centrados en las necesidades reales de la comunidad, generar nuevos conocimientos a través de la investigación y permitir a los educandos desarrollar prácticas académicas que contribuyen a la formación del futuro profesional.

Finalmente, el plan de marketing ayuda a las perspectivas de la Corporación, que se ajusta a lo exigido por la ley e incluso va más allá al centrarse en lo que los aliados esperan para que aumente el valor percibido y se optimice la imagen institucional.

\section{RECOMENDACIONES FINALES}

Entre las recomendaciones es fundamental que la CURN de el primer paso y sea pionera en el desarrollo de proyectos de investigación, apoyada por entidades como Colciencias, para que se genere un impacto social que redunde en be- 
neficios a la ciudad y se amplie el desarrollo de los mismos a la región e incluso a otras regiones del país.

Se debe trabajar con entidades que no optaron por el valor emocional positivo, con el propósito de cambiarles esa percepción, lo que redundará en mayores beneficios para las partes.

De otra parte, debe utilizar las herramientas propias: la página web de la institución, hacer canje con algún medio de comunicación, para socializar los convenios (los que considere relevantes), darlos a conocer, mostrar los resultados y los beneficios recibidos por ambas partes, lo que ayudará a generar un free press, trayendo como resultado el interés de nuevas entidades.

Vale aclarar que para tener el éxito asegurado en el desarrollo de este proyecto, es necesario realizar cada dos años la encuesta con los aliados, para verificar cómo han avanzado, que aspectos mejoraron, cuáles se deben revisar; apuntarle a dar cumplimiento a la mayoría de estrategias propuestas en el desarrollo de este trabajo, mantener una comunicación constante con los aliados, hacer seguimiento a los convenios y ejecutar correctamente el presupuesto.

\section{REFERENCIAS BIBLIOGRÁFICAS}

Arimany, Luis, (2011). Marketing Relacional: las percepciones. Consultado en abril de 2013. http:// www.luisarimany.com/marketing-relacional-las-percepciones

Barroso Castro, (1999). Marketing Relacional. Madrid, Editorial ESIC

CURN, Proyección Social Acuerdo 014 de 2009.

Fernández Morales, Antonio. (2002). Tamaños muéstrales: Instrumentos interactivos online para la formación estadísticas en el Sector Turístico. Málaga.

Gary Hamel, (1996). Strategy as Revolution, Harvard Business Review.

Kotler y Armstrong. (2003). Fundamentos de Marketing, Prentice Hall. 6ta. Edición.

La Investigación en valor percibido desde el Marketing. Tomado de: http://redalyc.uaemex.mx/ pdf/818/81803103.pdf

La Percepción. Consultado abril 2013 http://www.um.es/docencia/pguardio/documentos/percepcion. pdf

Mark Autor y David M. (1996). Estadística Básica en Administración Conceptos y Aplicaciones.

Mars, (2003). El marketing relacional: Kotler en el marketing. Consultado en abril 2013. http:// www.marsdd.com/articles/relationship-marketing-kotler-on-marketing.

Muñíz González, Rafael. Marketing en el siglo XXI. Consultado en Abril de 2013. http://www. marketing-xxi.com/etapas-del-plan-de-marketing-136.htm

Nuñez Roman, María de la Luz. (2003). Alianzas estratégicas: la mejor opción para su empresa. Consultado en Marzo de 2013. http://www.liderempresarial.com/num103/7.php

Porter, Michael, (1980). Competitive Strategy. The Free Press. New York 
Ruiz-Molina, María Eugenia. (2009). Valor percibido, actitud y lealtad del cliente en el comercio minorista. Consulta realizada en abril de 2013. http://ubr.universia.net/pdfs_web/UBR0012009102. pdf.

Sweeney, J.C.; Soutar, G.N. (2001) Consumer perceived value: the development of a multiple item scale, Journal of Retailing. Consultado en abril de 2013

Thompson Ivan. (2006). El plan de Marketing. Consultado en abril de 2013. http://www.marketingfree.com/articulos/plan-marketing.html

Vincenty Colon Rose. Figueroa Iglesias Nicol. (2011). Muestreo por Conveniencia. Consultado en Abril de 2013. http://www.slideshare.net/selene1524/muestreo-por-conveniencia 